\title{
Assessment of Data Quality on Expanded Programme on Immunization in Ghana: The Case of New Juaben Municipality
}

\section{Mavis Adamki ${ }^{1}$, Dominic Asamoah ${ }^{1}$ and Kwabena Riverson ${ }^{2 *}$}

${ }^{1}$ Department of Computer Science, Nkrumah University of Science \& Technology, KNUST, Kumasi, Ghana

${ }^{2}$ CSIR-Institute of Industrial Research, Accra, Ghana

\begin{abstract}
Organizations of all kinds make decisions based on the data they have at their disposal. In healthcare reporting, data quality and consistency are critical to ensuring patient safety and communicating health service delivery. Quality data provides accurate and timely information to manage services. It also provides good information to manage service effectiveness as well as aids to prioritize and ensure the best use of resources. The main objective of the study was to improve on data quality on expanded programme on immunization (EPI) in the New Juaben Municipality. The work aimed at improving the accuracy of reported number of vaccinations at vaccination delivery sites as well as assesses the completeness of data that was provided.
\end{abstract}

A descriptive cross-sectional study was employed. The study involved structured observation of tallied data from EPI tally books from the eight health sub-districts in the Municipality. Purposive sampling was used for this study. Data Quality Self-Assessment Tool (DQS) was the main instrument used in presenting and analyzing the accuracy and discrepancy ratios of the data. The result demonstrated discrepancies in tallied data at the vaccination delivery sites, facility summary report and report submitted to the Municipal Health Directorate. In 2011, there was 2674 over reported data to the district level while 2824 over reported data was recorded in 2012 from the eight (8) health facilities used for the study. It was observed that less importance was attached to data capture at some health facilities in the sub-districts. It was also ascertained that data storage and retrieval was very poor in some facilities visited. There is therefore the need for regular monitoring in the sub-districts' RCHs and Health Centers in the New Juaben Municipality of Ghana to correct the mistakes.

Keywords: Health care; Immunization; World Health Organization; Expandable programme on immunization (EPI); Data quality

\section{Introduction}

Immunization is among the very important public health interventions for preventing and reducing global child morbidity and mortality. The global effort to use vaccination as a public health intervention began in 1974 when the World Health Organization (WHO) launched the Expanded Programme on Immunization (EPI). There are often discrepancies between tallied data at the vaccination delivery sites and reported data from the same vaccination sites to the Municipal Health Directorate. This has necessitated the need for the research since high coverage of EPI and MDG 4 cannot be achieved without quality data. EPI coverage has to be improved but this cannot also be achieved without quality data or strengthening of the data management system. The New Juaben Municipality is the smallest of the 26 districts in the region and houses the Regional Capital (Table 1).

\section{Problem statement}

The weak health information system support within the subdistricts is one of the obstacles to effective and efficient management in the health service delivery. At the level of operation where service delivery is the main task, there are problems with data capture, analysis, storage and utilization of health service information for decision making. In EPI, tally data (recounted data) is usually different from summary data at the facility level and also that submitted to the District Health Directorate. This research would assess the quality aspect of EPI data generated in the municipality.

\section{Main objective}

To improve on data quality on Expanded Programme on Immunization (EPI) in the New Juaben Municipality.

\section{Specific objectives}

To determine the accuracy of reported number of vaccinations at vaccination delivery sites.

To assess the completeness of data.

To assess data storage and retrieval of data for action.

\section{Conceptual framework}

The framework above (Figure 1) explains most likely factors that lead to good EPI data quality. These include complete recording and documentation in EPI tally books, availability of EPI tally books and monthly reporting formats, availability of computers for data capture and analysis, availability of proper filing system and availability of patient registers. EPI tally books are very important since services provided in health facilities are to be documented for analysis as well as for future reference. The registers serve as source documents where summary reports are generated. The non existence of these registers implies that services were not provided. The correlation between the existences of these registers indicates whether or not data is manufactured at fake health facilities. Records keeping are one of the critical components of quality data. Good records keeping make data

*Corresponding author: Kwabena Riverson, CSIR-Institute of Industrial Research, Accra, Ghana, Tel: +233-30-2500195; E-mail: dr.riverson@csir-iir.org and dr.riverson@gmail.com

Received March 13, 2015; Accepted July 22, 2015; Published July 29, 2015

Citation: Adamki M, Asamoah D, Riverson K (2015) Assessment of Data Quality on Expanded Programme on Immunization in Ghana: The Case of New Juaben Municipality. J Health Med Informat 6: 196. doi:10.4172/2157-7420.1000196

Copyright: ( 92015 Adamki M, et al. This is an open-access article distributed under the terms of the Creative Commons Attribution License, which permits unrestricted use, distribution, and reproduction in any medium, provided the original author and source are credited. 
Citation: Adamki M, Asamoah D, Riverson K (2015) Assessment of Data Quality on Expanded Programme on Immunization in Ghana: The Case of New Juaben Municipality. J Health Med Informat 6: 196. doi:10.4172/2157-7420.1000196

Page 2 of 9

\begin{tabular}{|c|c|c|c|c|}
\hline NO. & Sub District & Number Of Recognised Communities & Population \\
\hline 1. & Jumapo & 13 & 13,361 \\
\hline 2. & Oyoko & 9 & 12,026 \\
\hline 3. & Effiduase & 11 & 16,234 \\
\hline 4. & Asokore /Akwadum & 20 & 20,828 & 6.5 \\
\hline 5. & Koforidua & 12 & 41,259 & 10.9 \\
\hline 6. & Zongo & 9 & 21.8 & 18,988 \\
\hline 7. & Adweso & 38 & 47,311 & 21,518 \\
\hline 8. & Old Estate / Densuano & 25 & 19.8 \\
\hline
\end{tabular}

Source: New Juaben Municipal Health Directorate, 2013

Table 1: Population Distribution by Health Sub Municipals

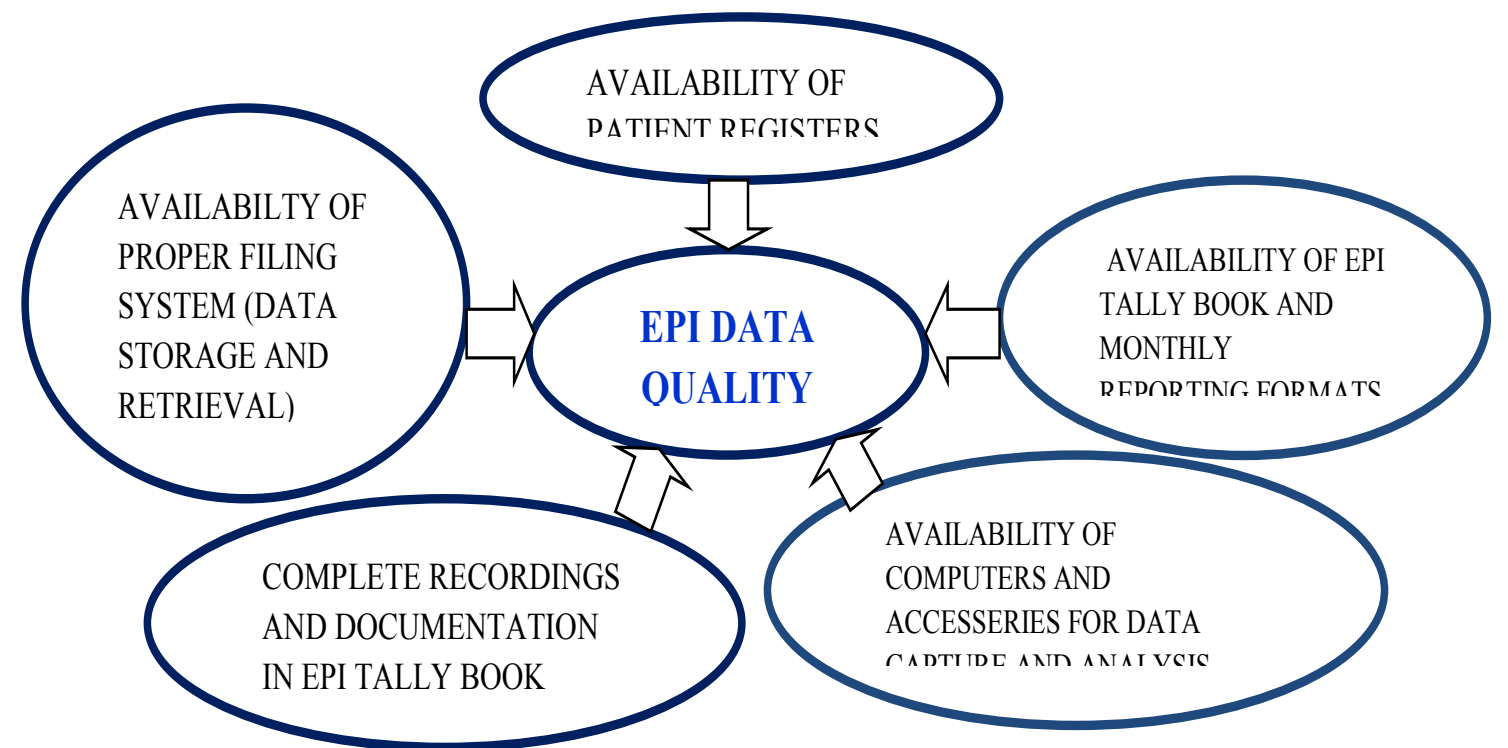

Figure 1: Conceptual framework on factors that result in good EPI data quality.

accessible at any point in time for decision making at all levels. It is worth mentioned that all patients' registers, tally books and all source data are kept well for continuity of care and for future references. If the source data (registers, tally books, patient folders) are not kept well, it becomes very difficult to make accurate decision as and when it is needed.

\section{Review of Literature}

\section{Introduction}

Immunization is a vital mechanism for controlling as well as eradicating infectious disease and in order to achieve this aim there should be quality data to aid in knowing the increasing coverage and decreasing drop-out rates. Quality data is one of the major problems facing the New Juaben municipality. In relation to EPI, children vaccinated are not tallied correctly and summary data do not always tally with reported data. The old EPI tally books are mishandled and misplaced and others. These have necessitated the need to research into this area to diagnose all associated problems and address them.

Data: Data is defined as abstract representations of selected characteristics of real world objects, events and concepts expressed and understood through explicitly definable conventions related to their meaning, collection and storage. The key to making decision about fitness for use is, knowing what the data is intended to represent and how they affect their representation [1].

Data quality: Data quality is the degree to which data meet the specific needs of specific customers. One customer may find data to be of high quality while another finds the same data to be of low quality [2].

Impacts of poor quality data: Poor quality data can imply a multitude of negative consequences in a company. To start with, poor quality data that is not identified and corrected can have significantly negative economic and social impacts on an organization $[3,4]$. The implications of poor quality data carry negative effects to business users through; less customer satisfaction, increased running costs, inefficient decision-making processes, lower performance and lowered employee job satisfaction [5-7].

Data quality assessment: There are three (3) categories of processes that cause data problems; Data processing, Data cleansing and Data purging. The quality of the data after conversion is directly proportional to the amount of time spent to analyze and profile the data and uncover the true data contet. Production of high quality information depends on the assessment of data quality [8].

Data analysis: Data analysis is a body of methods that help to describe facts, detect patterns, develop explanations, and test hypotheses. It is used in all of the sciences. It is used in business, in 
administration, and in policy. Analysis from data may reveal health trends and spark reaction from national health agencies to intervene before an epidemic begins [9-11].

Discrepancies and accuracy ratio: The immunization Data Quality Self-assessment (DQS) methodology was created by the World Health Organization (WHO) to evaluate the different aspects of the immunization monitoring system. The first DQS took place in Costa Rica from $3^{\text {rd }}$ to $12^{\text {th }}$ November 2005.

The Accuracy ratio (Data verification factor) is the main quantitative measure of data accuracy. It is the ratio between the number of vaccinations verified or recounted from a source (tally book) at one level (numerator), compared to the number of vaccinations reported by that level (facility summary report) to higher levels (denominator). This ratio gives the proportion of reported numbers that could be verified and it is expressed as a percent. If the accuracy ratio is less than $(<) 100$ percent $(\%)$ then the data is over reported [12-14]. On the other hand, if the accuracy ratio is greater than $(>) 100 \%$, then it is under reported.

\section{Methodology}

\section{Introduction}

This chapter outlines the various methods and materials that were used to achieve the study objectives. Both primary and secondary data were used as sources of information from both the vaccination delivery sites and the New Juaben Municipal Health Directorate, Koforidua. It also focuses on the strategies used in sampling and collecting data and how the data will be analyzed. In addition, data was entered into the DQS toolbox for analysis. Tables, frequencies and proportions were used to present the data.

Study sites: The study was carried out in the Reproductive and Child Health units (RCHs compound) and Health Centers in the New Juaben Municipality in the Eastern Region of Ghana. The Municipality has eight sub-districts RCHs and Health Centers represented in Table 1 above.

Research design: The study was descriptive cross sectional in content.

Study population: All the eight (8) sub-districts were visited for the research.

Sample size: Eight (8) health facilities were selected for the research. These facilities are the main eight sub-districts in the municipality. The study compared tallied data in the EPI tally registers at the vaccination delivery sites to facility summary report as well as to reported data at the Municipal Health Directorate to check for discrepancies.

Sampling procedure: Purposive sampling was used to assess data from all the eight sub-districts health facilities. The reason for adopting this sampling procedure was to identify any discrepancies in EPI tally books and reasons accounting for the unreliable data.

Data collection technique: The data quality self-assessment tool (DQS) was used to compare facility tallied data with reported data to check for discrepancies. In addition, an observational checklist was used to check for how data was captured, stored, retrieved, analyzed and utilize in the facility. Moreover, the study relied on vials using existing data from the district health directorate information unit. Data was obtained primarily from the EPI register books which specified number of vials given. This was collated by the Midwives in the various sub-districts at the end of every month and submitted to the Municipal
Health Directorate (MHD) in a form of hardcopies since they lack computers.

During the process, tallies from EPI tally books were compared with summary data at the facility and the municipal health directorate using the observation technique. Tallies in EPI books and summaries collated were observed and verified to check for discrepancies using the DQS data collection tool (Table 2).

Instrument for data collection: The data quality self-assessment tool (DQS), Table 3 below was used to review EPI records from 2011 to 2012. Tallied data (recounted data) was compared to summary report at the facility and later cross checked with the District summary report. The daily vaccinations administered in the EPI tally books were recorded into an EPI data collection tool. The aggregates for each month per antigen were then entered into the DQS tool which generated accuracy ratio (verification factor) and Discrepancy rate per health unit. This exercise is vital because it also provides an opportunity to evaluate coverage data accuracy and correct it.

An observational check list was also employed to assess data quality issues in the various health facilities. The observational check list covered areas such as data capture, storage and retrieval and data utilization.

Data processing and analysis: The DQS Tool was used in the quantitative data entry, processing and analysis. Data was entered into the DQS tool which generated accuracy ratio (verification factor) and Discrepancy rate per health unit and antigen. The DQS has the period of which data is collected by month thus January to December with its corresponding antigen that is assessed. When these indicators on the DQS are completely filled, the tool will automatically generate accuracy ratio represented on the tool as ' $\mathrm{VF}$ ' and discrepancy levels which is also indicated as 'discrepancy'.

Epi-Info software was also used to design a template of which the observational check list was entered. Statistical analysis was run using analysis command of the Epi-Info software.

Field observation: Each of the eight (8) sub-districts were visited

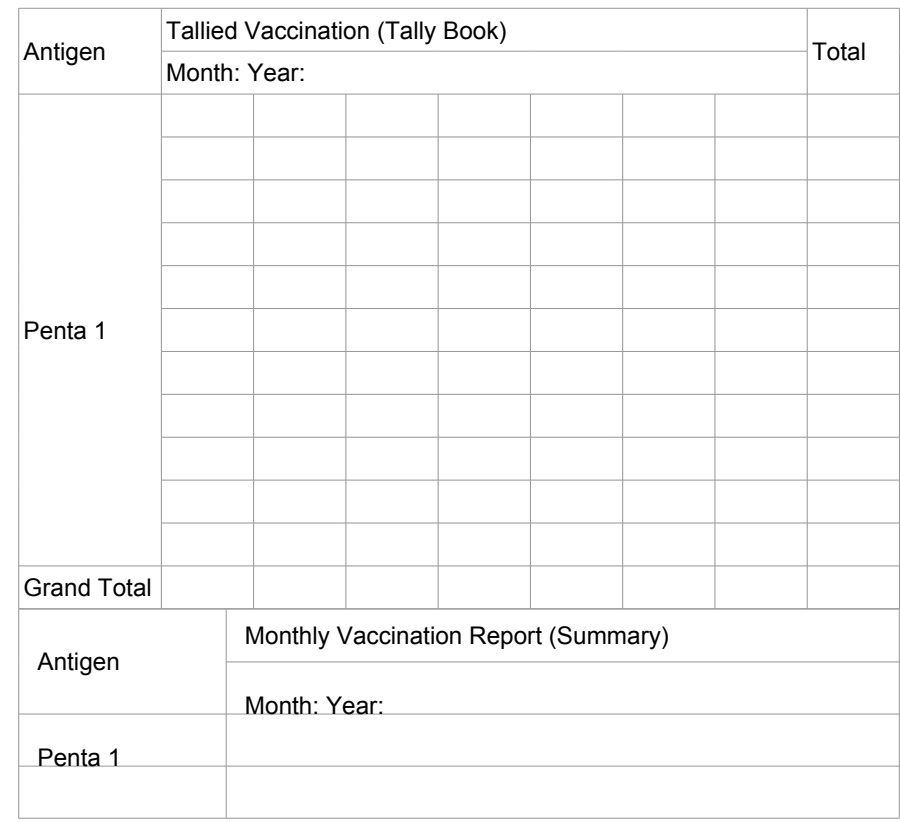

Table 2: DQS Data Collection Tool for One Antigen 


\begin{tabular}{|c|c|c|c|c|c|c|c|c|c|c|c|c|c|c|}
\hline Antigen & Jan & Feb & Mar & Apr & May & Jun & Jul & Aug & Sep & Oct & Nov & Dec & Total & \\
\hline Health Unit 4 & DEN & ANO & & & & & VF: & ND & & Disc & & ND & & \\
\hline Tally Sheet & - & - & - & - & - & - & - & - & - & - & - & - & - & 12 \\
\hline Summary Sheet & - & - & - & - & - & - & - & - & - & - & - & - & - & 12 \\
\hline Reported To District Level & - & - & - & - & - & - & - & - & - & - & - & - & - & 12 \\
\hline Recounted Data(Tally Sheet) & 0 & 0 & 0 & 0 & 0 & 0 & 0 & 0 & 0 & 0 & 0 & 0 & - & \\
\hline $\begin{array}{l}\text { Reported Data(Summary } \\
\text { Report) }\end{array}$ & 0 & 0 & 0 & 0 & 0 & 0 & 0 & 0 & 0 & 0 & 0 & 0 & - & \\
\hline Discrepancies & 0 & 0 & 0 & 0 & 0 & 0 & 0 & 0 & 0 & 0 & 0 & 0 & - & \\
\hline
\end{tabular}

Table 3: DQS Toolbox for a Single Health Facility

to check for their previous EPI vaccination registers, copies of previous summary reports, data completeness in the EPI registers, graphs showing trends of EPI coverage, EPI monitoring charts, reports from data validation team and finally how data was stored and retrieved in the facility.

Delimitation of the study: The study was limited to only New Juaben Municipality. The target for the study was only children below 1 year and pregnant women. Only Penta 1, Penta 3, Measles and TT2+ for pregnant women were assessed due to time constraints. Some health facilities could not produce primary source data (EPI tally book) for some of the periods under study. This will some way affect the analysis of data collected. Some health facilities were visited more than twice to complete data collection due to the volume of data to be assessed and with this, time and transport was a limitation.

Ethical issues: Permission was sought from the Municipal Health Directorate before the study was carried out. Upon reaching the health facilities, permission was again sought from the in-charges (Heads) before embarking on the exercise. The study also acknowledged all other sources of information used in reviewing literature thereby making it worth reading and source of information for future studies.

\section{Analysis and Findings of Data}

\section{Introduction}

The analysis focused on the accuracy and discrepancies of data in the eight (8) health facilities. Tallied data in EPI tally books at the vaccination delivery sites and summary report were considered in this analysis. All tallies in the EPI tally books for PENTA 1, PENTA 3, Measles and TT2+ for the period of 2011 to 2012 were counted and compared with the facility summary report and report submitted to the MHD to check for discrepancies. Again, a comparative analysis was drawn between the periods from 2011 to 2012 to ascertain whether there was improvement in the quality of EPI data $[15,16]$. With this, the data gathered from the EPI tally books were entered into the DQS toolbox and analyzed with the aid of graphical and statistical tabulation. The use of observational checklist was also employed. In all, eight (8) observational checklists were used in the eight health facilities in the eight sub-municipals.

\section{Data quality validation on PENTA 1, PENTA 3, Measles and TT2+}

Figure 2 above shows that, Koforidua Poly-Clinic had a high discrepancy rate of $48 \%$ of PENTA 1 antigen, followed by Medical
Village which recorded 26\%. Akwadum RCH, Zongo health centre, and Jumapo health centre recorded discrepancy of $9 \%, 3 \%$ and $1 \%$ respectively. Effiduase RCH and Densuano RCH recorded 100\% accuracy ratio with no discrepancy in PENTA 1 antigen [17-20]. It must be noted that the higher the discrepancy rate, the less quality the data is. These discrepancies occurred as a result of wrong tabulation and additions of tallied data in the vaccination sheet. It was also observed that some health workers do not tally direct into tally books during immunization services but rather use piece of papers to document and later transcribe into the tally book. Errors are made during the transcription of data from pieces of papers used during the immunization sections into the tally books. Totals of $19 \%$ discrepancy and $81 \%$ accuracy were recorded in 2011 for PENTA 1 . It should be noted that if the discrepancy recorded is more than $10 \%$ then the data is not reliable for decision making and planning of immunization programme. It is worth mentioned that structures are put in place to validate captured data from these vaccination centers before submitting it to the next level.

Figure 3 depicts that Jumapo Health Center had a high discrepancy rate of $77 \%$ of PENTA 1 antigen followed by Koforidua Poly-Clinic (44\%) and Densuano RCH (11\%). Effiduase RCH, Zongo Health Center, and Oyoko Health Center had a discrepancy level of $4 \%$, $2 \%$, and $3 \%$ respectively. Akwadum $\mathrm{RCH}$ and Medical Village $\mathrm{RCH}$ recorded $1 \%$ discrepancy level each. It must be noted that the higher the discrepancy rates the less quality the data is. Although Medical Village RCH had $101 \%$ accuracy ratio, there was $1 \%$ discrepancy rate recorded for PENTA 1 antigen which indicates under reporting. None of the health facilities visited had $100 \%$ accuracy ratio (AR) with no discrepancy in PENTA 1 antigen [21].

Making comparative analysis between accuracy ratio (AR) and the discrepancies of data in the reporting period of 2011 and 2012 of PENTA 1 antigen, it was noted that the accuracy ratio of PENTA 1 antigen in 2011 was $81 \%$ with $19 \%$ discrepancy level and still remains the same in 2012 although some health facilities recorded improvement of data quality with PENTA 1 antigen over the period of January to December, 2012. It was also observed that three (3) health facilities Akwadum Health Centre, Medical Village RCH, Zongo Health Centre recorded an improvement in data accuracy of the 2012 EPI data over 2011.

Figure 4 above depicts that Koforidua Poly-clinic had a high discrepancy rate of $55 \%$ of PENTA 3 antigen followed by Medical Village RCH (25\%) and Zongo Health Centre (5\%). Effiduase RCH, Oyoko Health Centre, Akwadum RCH and Densuano RCH recorded 


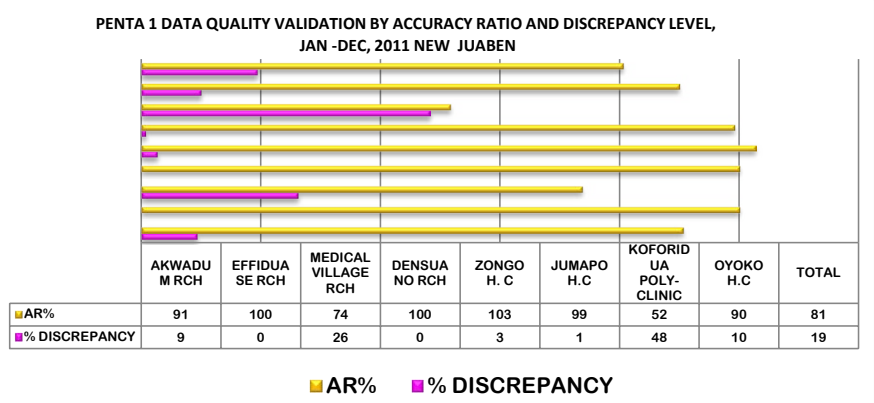

Figure 2: PENTA 1 Data Quality Validation by Accuracy Ratio and Discrepancy Level, Jan-Dec, 2011 New Juaben.

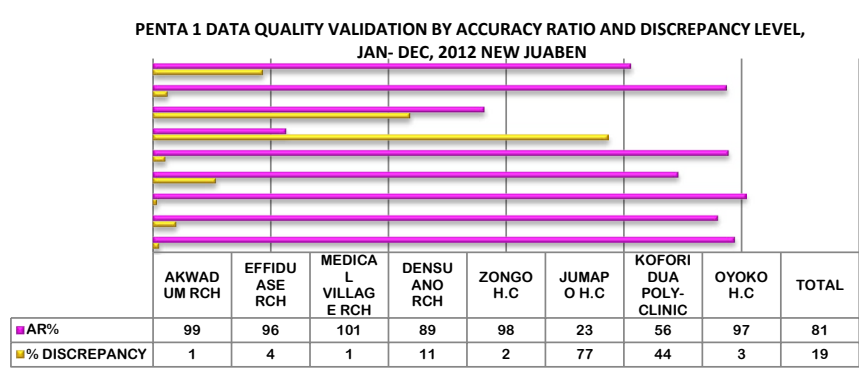

AR\% $\quad \square$ DISCREPANCY

Figure 3: PENTA 1 Data Quality validations by accuracy ratio and discrepancy level, Jan-Dec, 2012 New Juaben

discrepancy level of $4 \%, 3 \%, 2 \%$ and $1 \%$ respectively. Although Effiduase $\mathrm{RCH}$ had $104 \%$ accuracy ratio, there was $4 \%$ discrepancy rate recorded for PENTA 3 antigen which indicates under reporting. Only one health facility, Jumapo Health Center had 100\% accuracy ratio with no discrepancy in PENTA 3 antigen. It must be noted that the higher the discrepancy rate, the less quality the data is. Figure 4 show that PENTA 3 recorded $80 \%$ accuracy with $20 \%$ discrepancy for the period from January to December, 2011. As stated earlier, the accepted range of discrepancy level should be less than or equal to $10 \%$. This indicates that PENTA 3 data quality for 2011 was appalling and managers should address this challenge [22].

In Figure 5 above, only Oyoko Health Center recorded 100\% accuracy ratio. Jumapo Health Center recorded a very high discrepancy rate of $76 \%$ followed by Koforidua Poly-Clinic (41\%). These two facilities were not able to provide a complete EPI tally book for verification and this resulted in the high discrepancy. Moreover, apart from Oyoko Health Center, all the other facilities visited recorded less than $100 \%$ Accuracy (AR) for PENTA 3 Antigen which means there was over reporting.

A comparative analysis of figures 5 and 6 shows that data accuracy in 2011(80\%) of PENTA 3 antigen still remains the same in 2012. It was also noted that three (3) health facilities Medical Village $\mathrm{RCH}$, Koforidua Poly-Clinic and Oyoko Health Center recorded a slight improvement of data accuracy of PENTA 3 antigen in 2012.

In Figure 6 above, three (3) health facilities, Zongo Health Center, Jumapo Health Center and Effiduase RCH recorded 100\% accuracy ratio. Koforidua Poly-Clinic recorded the highest discrepancy rate of $50 \%$ followed by Medical Village $\mathrm{RCH}(25 \%)$. Generally the performance of data accuracy on the measles antigen was good with the exception of Koforidua Poly-clinic and Medical Village RCH as depicted by Figure 4.5 above. It was also ascertained in Figure 4.5 that $81 \%$ and $19 \%$ was recorded as accuracy ratio and discrepancy level for measles antigen from January to December, 2011. It can also be said that with the discrepancy ratio of $19 \%$, measles data for the year 2011 was of poor quality and not useful for any planning and decision making towards the immunization programme [23].

From Figure 7, none of the health facilities recorded $100 \%$ of accuracy ratio in measles antigen. One (1) Facility, Medical Village $\mathrm{RCH}$ recorded $101 \%$ of accuracy ratio with $1 \%$ of discrepancy which indicated under reporting while all the other facilities recorded less than $100 \%$ accuracy ratio showing over reporting. Two (2) health facilities, Jumapo Health Center and Koforidua Poly-Clinic recorded the highest discrepancy rate of $81 \%$ and $45 \%$ respectively

A comparative analysis of Figure 6 and Figure 7 depicts that, there was a 3\% improvement in measles data accuracy in 2012 over 2011. It was also ascertained that three (3) health facilities Medical Village $\mathrm{RCH}$, Oyoko Health Centre and Koforidua Poly-Clinic recorded improvement of data accuracy in 2012 over 2011. It was also noted that Koforidua Poly-Clinic recorded the highest discrepancy level of 50\% and $45 \%$ in 2011 and 2012 of measles data respectively.

From Figure 8 above, Koforidua Poly-Clinic had the highest discrepancy rate of 53\%, followed by Effiduase RCH (48\%), Oyoko Health Center recorded 38\%, Medical village recorded 31\%, Zongo and Jumapo Health Centers both recorded $1 \%$ discrepancy ratio each. Jumapo recorded $101 \%$ accuracy ratio (AR) with $1 \%$ of discrepancy ratio which indicated under reporting while all the other facilities recorded less than $100 \%$ accuracy ratio thus over reporting.

It was also noted that TT2+ recorded $59 \%$ and $41 \%$ of accuracy ratio and discrepancy level respectively from January to December, 2011. It must be noted that it is only the TT2+ antigen that recorded high discrepancy against accuracy. It can therefore be emphatically stated that, data quality for TT2+ was very low in this study. This is mainly issues with poor documentation on the part of health workers.

From Figure 9, none of the health facilities recorded $100 \%$ of accuracy ratio in TT2+ antigen. Two (2) facilities, Medical Village RCH and Zongo Health Center recorded 113\% accuracy ratio with $13 \%$ discrepancy and $104 \%$ accuracy ratio with $4 \%$ of discrepancies respectively which indicated under reporting. Jumapo Health Center had the highest discrepancy ratio of $77 \%$ with $23 \%$ accuracy. Effiduase $\mathrm{RCH}$ recorded $0 \%$ of accuracy ratio with $100 \%$ discrepancy and this indicates the highest over reported data for TT2+ antigen. It was also noted from figure 4.8 that TT2+ antigen recorded $60 \%$ and $40 \%$ accuracy ratio and discrepancy level respectively. It is noted that TT2+ had the poorest data quality as compared with the other antigens under study.

A comparative analysis of Figure 8 and Figure 9 depicts that, there was 1\% improvement in TT2+ data accuracy in 2012 over 2011. It was also ascertained that the general performance on the data capture or documentation on TT2+ antigen for 2012 was appalling in the Municipality although there was slight improvement over the 2011 data assessed.

Figure 10 above shows the tallies of all antigens against the summary reported to the District level. It is observed that TT2+ had the highest discrepancy followed by PENTA 1, Measles and PENTA 3 antigen. PENTA 1, PENTA 3, Measles, and TT2+ antigen recorded $617,397,509$, and 1151 over reported data in 2011 . This means that on 
Citation: Adamki M, Asamoah D, Riverson K (2015) Assessment of Data Quality on Expanded Programme on Immunization in Ghana: The Case of New Juaben Municipality. J Health Med Informat 6: 196. doi:10.4172/2157-7420.1000196

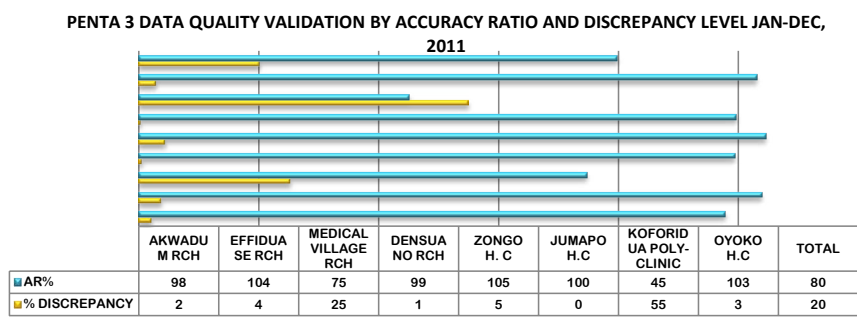

$\square \mathrm{AR} \% \quad \square \%$ DISCREPANCY

Figure 4: PENTA 3 Data Quality Validation by Accuracy Ratio and Discrepancy Level, Jan-Dec, 2011 New Juaben

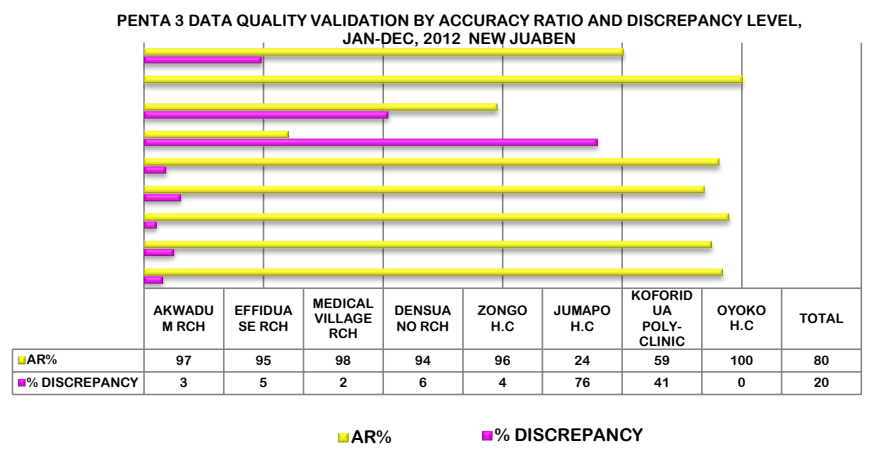

Figure 5: PENTA 3 Data Quality Validation by Accuracy Ratio and Discrepancy Level, Jan-Dec, 2012 New Juaben

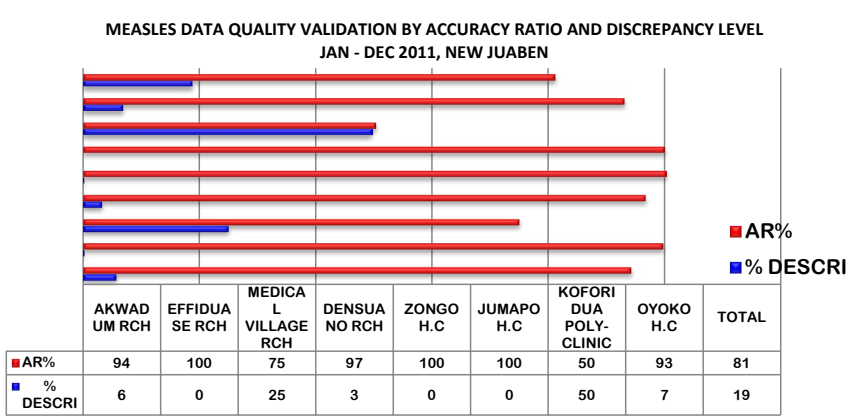

Figure 6: Measles Data Quality Validation by Accuracy Ratio and Discrepancy Level, Jan-Dec 2011, New Juaben

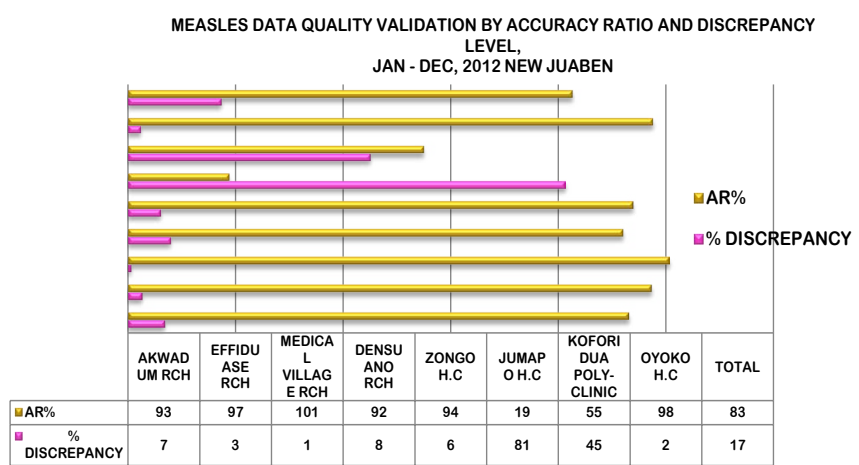

Figure 7: Measles Data Quality Validation by Accuracy Ratio and Discrepancy Level, Jan-Dec 2012 New Juaben

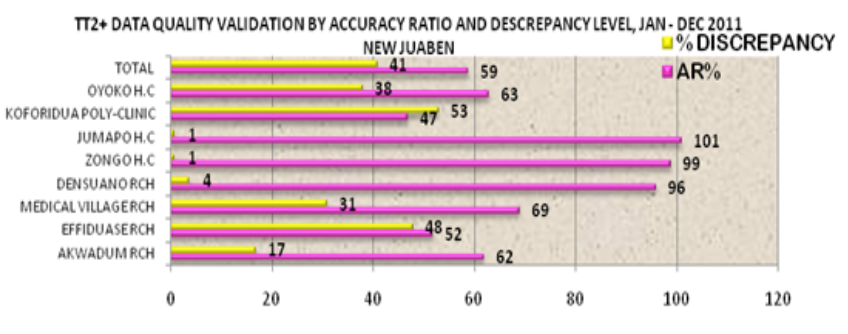

Figure 8: TT2+ Data Quality Validation by Accuracy Ratio and Discrepancy Level, Jan-Dec 2011 New Juaben

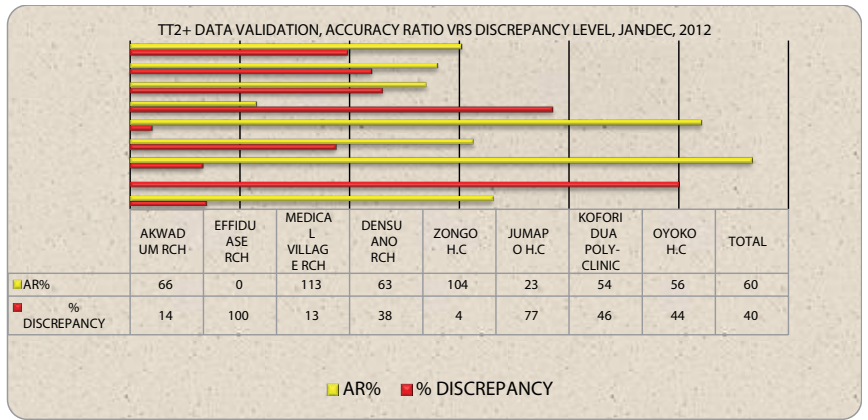

Figure 9: TT2+ Data Quality Validation, Accuracy Ratio vrs Discrepancy Level, Jan-Dec 2012 New Juaben

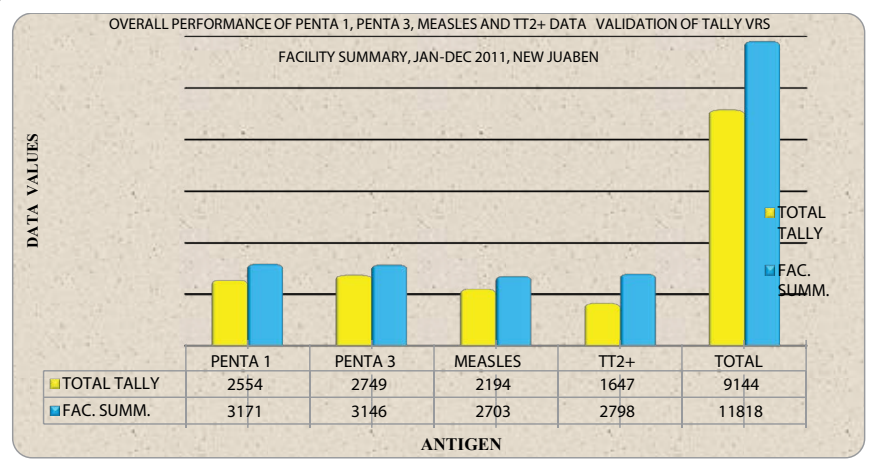

Figure 10: Overall Performance of PENTA 1, PENTA 3, Measles and TT2+ Data Validation of Total Tally vrs Facility Summary, Jan-Dec 2011 New Juaben.

the average 668 data was over reported per antigen to the Municipal Health Directorate. This depicts the low quality of data with respect to Expanded Programme on Immunization (EPI) where 2674 figures were over reported to the District, Regional and National Level from the eight health facilities assessed.

Figure 11 above shows the tallies of all antigens against the summary reported to the District level. Penta 1, Penta 3, Measles, and TT2+ recorded 590, 615, 479 and 1140 over reported data respectively to the municipal health directorate in 2012. It is observed that TT2+ had the highest discrepancy followed by PENTA 3, PENTA 1 and Measles Antigen. This depicts low quality of data with respect to Expanded Programme on Immunization (EPI) where 2824 figures were over reported to the District, Regional and National Level from the eight health facilities assessed. 


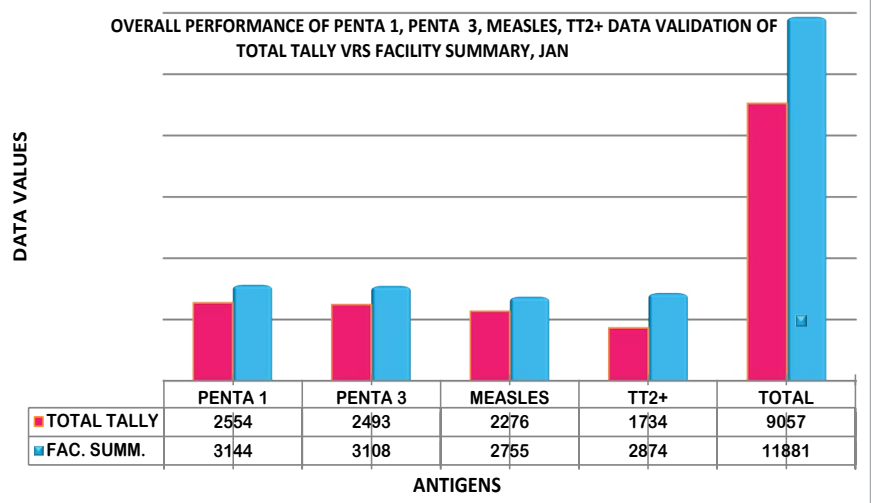

Figure 11: Overall Performance of PENTA 1, PENTA 3, Measles, TT2+ Data Validation of Total Tally vrs Facility Summary, Jan-Dec, 2012 New Juaben

Comparing Figure 10 and 11, it was noted that there was no improvement in data capture or documentation on EPI data in 2012 with the various antigen under study.This was greatly caused by the non existence of EPI tally books in three health facilities. Tally book for Jumapo Health Center from January to September 2012 could not be provided by the health facility. Koforidua Poly-Clinic also could not provide tally book for the period of March to July, 2012, and Medical Village RCH could also not provide tally book for Jan-March, 2011.

Field data were entered into DQS Toolbox to generate AR\% and \% DISCREPANCY figures. Moreover, the formula for generating the AR (\%) and \% DISCREPANCY is as follows

\section{AR $(\%)=$ Total Tallies/Facility Summary Data $\times 100$}

$\%$ DISCREPANCY $=100-\mathrm{AR}$.

If $\mathrm{AR}$ is $<100 \%$, data is over reported. If $\mathrm{AR}$ is $>100 \%$, data is under reported.

Table 4 illustrates the accuracy ratio (AR) and discrepancy ratio of the various antigen data. It is shown in Table 4 above that PENTA 3 antigen recorded the highest data accuracy of $87 \%$ with $13 \%$ discrepancy level while both PENTA 1 and Measles recorded $81 \%$ with $19 \%$ discrepancy. TT2 + antigen also recorded $59 \%$ of data accuracy with $41 \%$ discrepancy. The total data accuracy reported by these antigens from the eight (8) health facilities was $77 \%$ and $23 \%$ discrepancy rate.

Table 5 illustrates the accuracy ratio of the various antigen data. It is shown in Table 5 that Measles antigen recorded the highest data accuracy of $83 \%$ with $17 \%$ of discrepancy while PENTA 1, PENTA 3 , and TT2+ recorded $81 \%$ accuracy with $19 \%$ discrepancy, $80 \%$ accuracy ratio with $20 \%$ discrepancy, and $60 \%$ accuracy ratio with $40 \%$ discrepancy respectively. The total data accuracy ratio reported by these antigens from the eight (8) health facilities was $76 \%$ with $24 \%$ discrepancy.

\section{Analysis of the observational checklist}

Table 6 illustrates that $63 \%$ of the health facilities assessed had EPI tally books for the period under study while $37 \%$ of health facilities did not have. Non availability of the EPI tally book which implies inadequate poor records keeping can lead to poor EPI data quality. EPI tally books are very important since services provided in health facilities are to be documented for analysis as well as for future reference. The registers serve as source documents where summary reports are generated. The non-existence of these registers implies that services were not provided. The correlation between the existences of these registers indicates whether or not data is manufactured at fake health facilities.

Table 7 above illustrates that all health facilities assessed had copies of summary sheet $(100 \%)$ at their level which was encouraging. Good records keeping make data accessible at any point in time for decision making at all levels. It is worth mentioned that all patients' registers, tally books and all source data are kept well for continuity of care and for future references. If the source data (registers, tally books, patient folders) are not kept well, it becomes very difficult to make accurate decision as and when it is needed.

Data from Table 8 shows that $50 \%$ of health facilities assessed did not have complete tally books while $50 \%$ had their tally books complete.

From the review of the literature, data quality refers to data that is accurate, valid, reliable, relevant, legible, complete and available in a timely manner to decision makers for healthcare delivery and planning purposes. Complete data gives the right detail of information hence

\begin{tabular}{|c|c|c|}
\hline ANTIGEN & AR\% & $\%$ DISCREPANCY \\
\hline PENTA 1 & 81 & 19 \\
\hline PENTA 3 & 87 & 13 \\
\hline MEASLES & 81 & 19 \\
\hline TT2+ & 59 & 41 \\
\hline TOTAL & 77 & 23 \\
\hline
\end{tabular}

Table 4: Overall Performance Of PENTA 1, PENTA 3, Measles, TT2+ Antigen By Accuracy Ratio And Discrepancy Level Jan-Dec, 2011

\begin{tabular}{|c|c|c|}
\hline ANTIGEN & AR\% & \% DISCREPANCY \\
\hline PENTA 1 & 81 & 19 \\
\hline PENTA 3 & 80 & 20 \\
\hline MEASLES & 83 & 17 \\
\hline TT2+ & 60 & 40 \\
\hline TOTAL & 76 & 24 \\
\hline
\end{tabular}

Source: Field Data Were Entered Into DQS Toolbox To Generate AR\% \& \% DISCREPANCY Figures

Table 5: Overall Performance Of PENTA 1, PENTA 3, Measles, TT2+ Antigen By Accuracy Ratio And Discrepancy Level, Jan-Dec, 2012

\begin{tabular}{|c|c|c|}
\hline Observation & Frequency & Percent \\
\hline Yes & 5 & 63 \\
\hline No & 3 & 37 \\
\hline Total & 8 & 100 \\
\hline
\end{tabular}

Source: Field Data May 2013

Table 6: Does Health Facility Have EPI Tally Book For The Period Under Study (Jan-Dec, 2011, 2012).

\begin{tabular}{|l|l|l|}
\hline Observation & Frequency & Percent \\
\hline Yes & 8 & 100 \\
\hline Total & 8 & 100 \\
\hline
\end{tabular}

Source: Field Data May 2013

Table 7: Existence Of Monthly Summary Sheet At Health Facility For The Period Under Study (Jan-Dec, 2011, 2012) 
making analysis and interpretation easier and arriving at practical solutions to address challenges.

Data from Table 9 shows that $25 \%$ of health facilities assessed had excellent data storage/ retrieval (filing system) while 12.5\%, 25\% and $37.5 \%$ had very good, good and poor filing system respectively.

There are many ways by which EPI data can be stored in the health facilities for easy retrieval in decision making. Good records keeping make data accessible at any point in time for decision making at all levels. It is worth mentioned that all patients' registers, tally books and all source data are kept well for continuity of care and for future references.

Data from Table 10 shows clearly that all health facilities assessed had EPI monitoring chart for the current year pasted. Table 11 illustrates that $50 \%$ of health facilities assessed have their EPI monitoring chart of the previous month updated while $50 \%$ of health facilities have not updated their EPI monitoring chart.

\section{Summary of Findings, Conclusions and Recommendation Introduction}

The summary of findings of the research, conclusions drawn based on the findings and recommendations made are discussed in this chapter.

\section{Data completeness}

Data completeness deals with whether or not the right detail of information is provided. It was observed in table 4.5 that $50 \%$ of health facilities had incomplete tally books. This is likely to affect data quality in the sense that the right level of details of information that should be provided is not available for good decision making and planning purposes. For example estimates for vaccines to be used for vaccinations are base on the documentations in the tally books hence vaccines would be over budgeted or under budgeted for EPI programmes.

\section{Data storage and retrieval (filing system)}

Stored data are of use only if information can be retrieved quickly in an understandable form. Having a large quantity of information put together in common file might cause difficult access to any particular piece of information. That was the situation in our health facilities. It was being noted that a large quantity of different data is kept together in health facilities and this makes it difficult for data retrieval when decisions are to be made.

It was also observed that all health facilities assessed did not have electronic data storage system but relies on the manual system of data storage and retrieval. It was again ascertained that data storage and retrieval was very poor in some facilities especially Koforidua PolyClinic, Jumapo Health Center and Medical village RCH.

\section{Conclusion}

Judging from the findings of the research, the conclusion drawn was that the quality of data with respect to the Expanded Programme on Immunization (EPI) is generally poor.

As indicated earlier on, source data (tally books) from three (3) health facilities could not be produced for verification and this resulted in wide margins of over reported cases. The poor data quality can also be attributed to poor handling of data in these health facilities.

\begin{tabular}{|c|c|c|}
\hline Observation & Frequency & Percent \\
\hline Yes & 4 & 50 \\
\hline No & 4 & 50 \\
\hline Total & 8 & 100 \\
\hline
\end{tabular}

Source: Field Data May 2013

Table 8: Does All Health Facilities Have Completed Tally Books (Where Complete Means All Indicators in Tally Book Such As Number of Vaccine Used, Number of Children Vaccinated Are Documented)

\begin{tabular}{|c|c|c|}
\hline Observation & Frequency & Percent \\
\hline Excellent & 2 & 25 \\
\hline Very Good & 1 & 12.5 \\
\hline Good & 2 & 25 \\
\hline Poor & 3 & 37.5 \\
\hline Total & 8 & 100 \\
\hline
\end{tabular}

Source: Field Data May 2013

Table 9: What Is The Data Storage/Retrieval Situation (Filing System) In The Health Facility?

\begin{tabular}{|c|c|c|}
\hline Observation & Frequency & Percent \\
\hline Yes & 8 & 100 \\
\hline Total & 8 & 100 \\
\hline
\end{tabular}

Source: Field Data May 2013

Table 10: Does Facility Has EPI Monitoring Chart for the Current Year (2013) Pasted (On Facility Notice Board).

\begin{tabular}{|c|c|c|}
\hline Observation & Frequency & Percent \\
\hline Yes & 4 & 50 \\
\hline No & 4 & 50 \\
\hline Total & 8 & 100 \\
\hline
\end{tabular}

Source: Field Data May 2013

Table 11: If Yes, Is The Previous Month Updated?

\section{Recommendations}

In analyzing the data and findings of the research work, some problems had been identified and as a result the following recommendations and suggestions have been provided;

1. EPI tallies must be done as soon as vaccines are administered to avoid distortion of data in the vaccination delivery sites.

2. Data audit teams should be instituted at all facilities to validate monthly and quarterly data before submission to the next level.

3. The Municipal Health Directorate and Sub-municipal heads should intensify Facilitative supervisory visits at all levels and orient health staff involved in data collection on the importance of quality data.

4. The Municipal Health Directorate should provide cabinets, files, computers and accessories for health facilities to improve on the data storage and retrieval system in the Municipality.

5. Health facility heads or sub-municipal heads should make sure that daily summaries of children vaccinated and logistics used are documented in tally books to enhance accurate data recordings at the end of the month.

6. Data quality assessment should be ritualized in the routine monitoring and support visits to all health facilities.

7. Further research should be conducted into factors affecting poor data quality on expanded programme on immunization by the Municipal Health Directorate. 
Citation: Adamki M, Asamoah D, Riverson K (2015) Assessment of Data Quality on Expanded Programme on Immunization in Ghana: The Case of New Juaben Municipality. J Health Med Informat 6: 196. doi:10.4172/2157-7420.1000196

\section{References}

1. Sebastian-Coleman L (2013) Measuring Data Quality for On-going Improvement: A Data Assessment Framework (The Morgan Series). Elsevier, USA.

2. Redman TC (2001) Data Quality: The Field Guide. Boston: Digital Press, USA.

3. Ballou DP, Pazer HL (1995) Designing Information Systems to Optimize the Accuracy-timeliness Tradeoff. Information Systems Research 6: 51-72.

4. Wang Y, Wang RY (1996) Anchoring data quality dimensions in ontological foundations. Communications of the ACM 39: 86-95.

5. Kahn BK, Strong DM, Wang RY (2003) Information quality benchmarks: Product and service performance. Communications of the ACM 45: 184-192.

6. Lee WY, Pipino LL, Funk JD, Wang RY (2006) Journey to Data Quality. Massachusetts Institute of Technology, USA

7. Redman TC (1996) Data Quality for the Information Age. Norwood, MA: Artech House, USA.

8. Aiken PH, Davis KH (1996) Data reverse engineering. McGraw-Hill, New York, USA.

9. Ballou DP, Pazer HL (1985) Modeling data and process quality in multi-input multi-output information systems. Management Science 31: 150-162.

10. Batini C, Cappiello C, Francalanci C, Maurino A (2009) Methodologies for Data Quality Assessment and Improvement. ACM Computing Surveys 41.

11. Batini C, Scannapieco M (2006) Data Quality: Concepts, Methodologies and Techniques (Data Centric Systems and Applications)
12. Blasius J, Thiessen V (2012) Assessing the Quality of Survey Data (Research Methods for Social Scientists). SAGE. London.

13. Bos E, Batson A (2000) Using immunization coverage rates for monitoring health sector performance. Washington, DC: The World Bank, USA

14. Brackett MH (2000) Data Resource Quality: Turning Bad Habits into Good Practices. Englewood Cliffs, NJ: Addison Wesley Longman.

15. Brackett MH (1996) The Data Warehousing Challenge, Taming Data Chaos. New York: John Wiley \& Sons, USA.

16. Cassidy A (1998) A practical guide to Information Systems Strategies Planning $2^{\text {nd }}($ Edn). St. Lucie CRC Press LLC, USA.

17. GHS (2002) Ghana Expanded Programme On Immunization: Reducing Missed Opportunities.

18. Trostle M, Steinglass R, Fields R, Favin M, Ballou S (2003) Immunization Essential, A Practical guide 12: 61-85 \& 206-208.

19. Loshin D (2010) The Practitioner's Guide to Data Quality Improvement. Morgan Kaufmann, USA

20. Loshin D (2001) Enterprise Knowledge Management: The Data Quality Approach. San Francisco: Morgan Kaufmann, USA.

21. Municipal Health Directorate Koforidua. Annual Report 2011 and 2012

22. Olson JE (2003) The Accuracy Dimension (The Morgan Kaufmann Series in Data Management Systems). San Francisco. Morgan Kaufmann, USA.

23. Watts S, Shankaranarayanan AE, Even A (2009) Data quality assessment in context: A cognitive perspective. Decision Support Systems 48: 202-211. 